\title{
Konditionierung bei AML: Weniger intensiv tut's auch
}

Bei Patienten mit akuter myeloischer Leukämie (AML) ist eine intensive Konditionierung vor der allogenen Stammzelltransplantation offenbar nicht erforderlich, eine weniger intensive tut es auch.

In einer prospektiven deutschen Studie unterschieden sich krankheitsfreies und Gesamtüberleben nicht wesentlich. Im Rahmen der Studie erhielten 99 Patienten mit akuter myeloischer Leukämie in erster Komplettremission eine weniger intensive Konditionierung als bisher üblich und 96 Patienten die Standardkonditionierung. Das bedeutet, die AML-Patienten erhielten wenige Tage vor der Transplantation eine Ganzkörperbestrahlung mit vier Strahlendosen à 2 Gy plus 150 $\mathrm{mg} / \mathrm{m}^{2}$ Fludarabin oder mit sechs Dosen à 2 Gy plus $120 \mathrm{mg} / \mathrm{kg}$ Körpergewicht Cyclophosphamid. Die Patienten waren zwischen 18 und 60 Jahre alt. Zur Prophylaxe einer Graft-versus-host-Krank- heit wurden alle mit Ciclosporin plus Methotrexat behandelt. Primärer Endpunkt der Studie war Mortalität, die nicht Folge eines Rezidivs ist.

Drei Jahre nach der allogenen Stammzelltransplantation lag die Mortalität in der Gruppe der Patienten mit weniger intensiver Konditionierung bei $13 \%$, in der Gruppe mit Standardkonditionierung bei $18 \%$ - mit $\mathrm{p}=0,22$ kein statistisch signifikanter Unterschied. Auch bei der Inzidenz der Rezidive und der kumulativen Inzidenz der Rezidive nach drei Jahren gab es keinen signifikanten Unterschied zwischen den beiden Gruppen. Betrachtet man nur das erste Jahr nach Konditionierung und Transplantation tritt der Vorteil der weniger intensiven Vorbehandlung noch deutlicher zutage. Denn aufgrund der geringeren Nebenwirkungen sterben im ersten, kritischen Jahr der Therapie weniger als $10 \%$ der Patienten an den Folgen der Behandlung, wie Martin Bornhäuser von der Universitätsklinik Dresden in einer Mitteilung des Klinikums zitiert wird. In der Gruppe der Patienten mit Standardkonditionierung seien es dagegen fast $20 \%$.

Die Hämatologen erinnern daran, dass viele Daten aus Studien und Registern vermuten lassen, dass selbst die nicht myeloablative Konditionierung Leukämien heilen kann, was auf den antileukämischen Effekt von Spenderzellen der nachfolgenden Transplantation zurückzuführen ist.

Peter Leiner

Bornhäuser $\mathrm{M}$ et al. Reduced-intensity conditioning versus standard conditioning before allogeneic haemopoietic cell transplantation in patients with acute myeloid leukaemia in first complete remission: a prospective, open-label randomised phase 3 trial. Lancet Oncol. 2012; 13(10):1035-44.

\section{Tumorresektion bei Grad-II-Gliomen verlängert das Leben}

Im Rahmen einer Studie werteten norwegische Neurochirurgen Daten von $153 \mathrm{~Pa}$ tienten aus, die mit Grad-II-Gliomen in zwei Kliniken behandelt wurden. Nach einer diagnostischen Biopsie lautete in der einen Klinik die Strategie „wait and scan“,

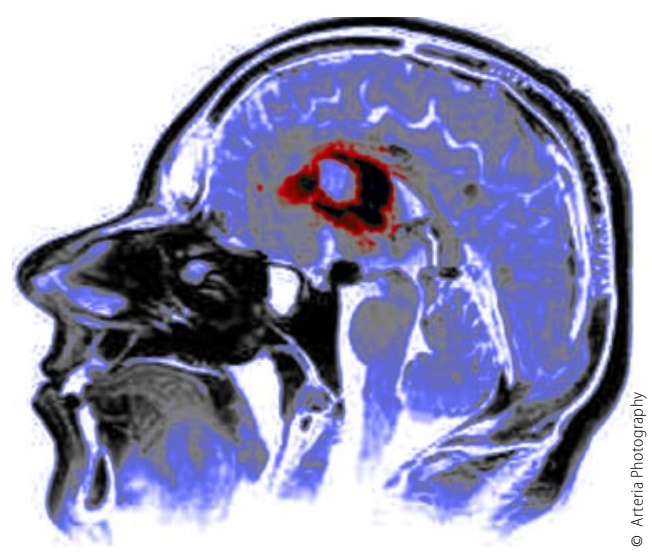

Auch bei Grad-II-Gliomen scheint die rasche Resektion von Vorteil. also abwarten sowie regelmäßig das Tumorwachstum per Bildgebung kontrollieren. Eine MRT-Aufnahme erfolgte drei und sechs Monate nach Diagnose, danach jährlich. Erst bei Tumorwachstum oder bei Zeichen einer erhöhten Malignität wie etwa neue Kontrastmittelanreicherungen - resezierten die Chirurgen den Tumor. In der zweiten Klinik kam ein neues ultraschallgestütztes Navigationssystem für die Hirn-Operation zum Einsatz, das die Risiken des Eingriffs minimieren soll. Auch Grad-II-Tumoren wurden meist gleich nach der Diagnose entfernt. Eine Auswertung der Daten über sieben Jahre zeigte, dass von den 87 Patienten mit früher Resektion nach fünf Jahren noch $74 \%$ lebten, in der Klinik mit „wait and scan“ waren es nur $60 \%$ von 66 Patienten. Zum Studienende nach sieben Jahren waren in der abwartenden Gruppe $52 \%$ der Patienten gegenüber $32 \%$ in der Gruppe mit früher Resektion gestorben. Auch waren mit „wait and scan“ maligne Transformationen häufiger als in der Resektionsgruppe (56 vs.37\%). Bei der Hälfte der Patienten in der „wait and scan“-Gruppe wurde im Studienverlauf der Tumor ebenfalls entfernt. Insgesamt waren bei sechs Patienten (9\%) dieser Gruppe gegenüber sieben (8\%) der Gruppe mit früher Resektion operationsbedingte Komplikationen aufgetreten. Oft hätten Ärzte und Patienten laut den Autoren Angst, die frühe Resektion verschlechtere die Lebensqualität. Der anfängliche Vorteil für die Lebensqualität beim Abwarten ginge aber aufgrund der häufigen Tumorprogression rasch verloren. Zudem scheint der Unterschied bei den Überlebensraten mit der Zeit immer größer zu werden: Lag diese Differenz nach fünf Jahren bei $14 \%$, so waren es nach sieben Jahren bereits $24 \%$. Die Forscher erklären sich den Nutzen einer frühen Biopsie schlicht damit, dass dann weniger Zellen übrig bleiben, die maligne transformieren können. Thomas Müller

Jakola AS et al. Comparison of a Strategy Favoring Early Surgical Resection vs a Strategy Favoring Watchful Waiting in Low-Grade. JAMA. 2012 Oct 25:1-8 [Epub ahead of print]. 